\title{
3D Image Segmentation of Deformable Objects with Shape-Appearance Joint Prior Models
}

\author{
Jing Yang $^{1}$ and James S. Duncan ${ }^{1,2}$ \\ 1 Departments of Electrical Engineering \\ 2 Diagnostic Radiology, Yale University, P.O. Box 208042 \\ New Haven CT 06520-8042, USA \\ $\{\mathrm{j} \cdot \mathrm{yang}, \mathrm{james}$.duncan\}@yale .edu
}

\begin{abstract}
We propose a novel method for 3D image segmentation, where a Bayesian formulation, based on joint prior knowledge of the shape and the image gray levels, along with information derived from the input image, is employed. Our method is motivated by the observation that the shape of the object and the gray level variation in an image have consistent relations that provide configurations and context that aid in segmentation. We define a Maximum A Posteriori(MAP) estimation model using the joint prior information of the shape and image gray levels to realize image segmentation. We introduce a representation for the joint density function of the object and the image gray level values, and define joint probability distribution over the variations of object shape and the gray levels contained in a set of training images. By estimating the MAP shape of the object, we formulate the shape-appearance model in terms of level set function as opposed to landmark points of the shape. We found the algorithm to be robust to noise, able to handle multidimensional data, and avoiding the need for point correspondences during the training phase. Results and validation from various experiments on 2D/3D medical images are demonstrated.
\end{abstract}

\section{Introduction}

The accurate segmentation and quantitative analysis of structures in an image is a fundamental issue in a variety of applications including medical image processing, computer vision and pattern recognition.

In the active contour approach (snakes) of Kass et al. (1987)) [1]), energy minimizing parametric contours with smoothness constraints are deformed according to the image data. Unlike level set implementations[2], the direct implementation of this energy model is not capable of handling topological changes of the evolving contour without explicit discrete pixel manipulations.

Image gray level based methods often face difficult challenges such as poor image contrast, noise, and missing or diffuse boundaries. Prior model based algorithms can often solve this problem. Staib and Duncan [3] incorporate global shape information into the segmentation process by using an elliptic Fourier decomposition of the boundary and placing a Gaussian prior on the Fourier 
coefficients. Leventon et al. [4] extend Caselles' geodesic active contours by incorporating shape information into the evolution process. Cootes et al. [5] find corresponding points across a set of training images and construct a statistical model of shape variation from the point positions.

Cootes et al. [6] use statistical models of shape and texture as deformable anatomical atlases. The modelled structure can be located by finding the parameters which minimize the difference between the synthesized model image and the target image in conjunction with the statistical model of the shape based on landmark points and texture. While the point correspondence problem needs to be taken care of and is difficult in 3D images.

Our work shares the observation that object to be detected and the gray level variation in an image have a consistent relation that can aid in the delineation. Our model is based on a MAP framework using the shape-appearance joint prior information. We introduce a representation for the shape-appearance joint density function and define the corresponding probability distribution. Formulating the segmentation as a MAP estimation of the shape of the object and modeling in terms of level set function, we compute the associated Euler-Lagrange equation. We avoid the need for point correspondences during the training phase by using level set representation of the shape. The contour evolves both according to the shape-appearance joint prior information and the image gray level information.

\section{Description of the Model}

\subsection{MAP Framework with Shape-Image Joint Prior}

The shape of an object is always dependent on the gray level variation in an image. Segmentation can be made easier if suitable models containing such relationship priors are available. A probabilistic formulation is a powerful approach to deformable models. Deformable models can be fit to the image data by finding the model shape parameters that maximize the posterior probability. Consider an image $I$ that has a shape $S$ of interest; a MAP framework can be used to realize image segmentation combining prior information and image information:

$$
\hat{S}=\arg \max _{S} p(S / I)=\arg \max _{S} p(S, I) / p(I)=\arg \max _{S} p\left(S, I=I_{0}\right)
$$

where $I_{0}$ is the given image. $p(S, I)$ is the joint density function of shape $S$ and image intensity $I$. It contains the shape prior information, the appearance prior information, as well as their relation.

\subsection{Shape-Image Joint Prior Model}

Instead of using a point model to represent the shape as was done in the pioneering work of Cootes[5][6], we choose level set[2] as our representation to build a model for the shape-image joint prior, and then define the joint probability density function in equation (1). 
Consider a training set of $n$ aligned images $\left\{I_{1}, I_{2}, \ldots, I_{n}\right\}$, with a shape of interest in each image. The surfaces of each of the $n$ shapes in the training set are embedded as the zero level set of $n$ separate higher dimensional level sets $\left\{\Psi_{1}, \Psi_{2}, \ldots, \Psi_{n}\right\}$ with negative distances inside and positive distances outside the object. Using the technique developed in [4], each of the $I_{i}$ and $\Psi_{i}$ is placed as a column vector with $N^{d}$ elements, where $d$ is the number of spatial dimensions and $N^{d}$ is the number of samples of each level set function or the number of pixels of each image. We can use vector $\left[\Psi_{i}^{T}, I_{i}^{T}\right]^{T}$ as the representation of the shape and intensity values. Thus, the corresponding training set is $\left\{\left[\Psi_{1}^{T}, I_{1}^{T}\right]^{T},\left[\Psi_{2}^{T}, I_{2}^{T}\right]^{T}, \ldots,\left[\Psi_{n}^{T}, I_{n}^{T}\right]^{T}\right\}$. Our goal is to build a shape-appearance model over the distribution of the level set function and intensity pair.

The mean and variance of the shape-intensity pair can be computed using Principal Component Analysis(PCA)[5]. The mean shape-intensity pair, $\overline{\left[\Psi^{T}, I^{T}\right]^{T}}=\frac{1}{n} \sum_{i=1}^{n}\left[\Psi_{i}^{T}, I_{i}^{T}\right]^{T}$, is subtracted from each $\left[\Psi_{i}^{T}, I_{i}^{T}\right]^{T}$ to create the deviation from the mean. Each such deviation is placed as a column vector in a $2 N^{d} \times n$ dimensional matrix $Q$. Using Singular Value Decomposition(SVD), $Q=U \Sigma V^{T} . U$ is a matrix whose column vectors represent the set of orthogonal modes of shape-intensity variation and $\Sigma$ is a diagonal matrix of corresponding singular values. An estimate of the shape-intensity pair $\left[\Psi^{T}, I^{T}\right]^{T}$ can be represented by $k$ principal components and a $k$ dimensional vector of coefficients(where $k<n), \alpha[5]$ :

$$
\widetilde{\left[\begin{array}{c}
\Psi \\
I
\end{array}\right]}=\overline{\left[\begin{array}{c}
\Psi \\
I
\end{array}\right]}+U_{k} \alpha
$$

Under the assumption of a Gaussian distribution of shape-intensity pair represented by $\alpha$, the joint probability of a certain shape $S$ and the related image intensity $I, p(S, I)$, can be represented by:

$$
p(\alpha)=\frac{1}{\sqrt{(2 \pi)^{k}\left|\Sigma_{k}\right|}} \exp \left[-\frac{1}{2} \alpha^{T} \Sigma_{k}^{-1} \alpha\right]
$$

Figure 1 shows a training set of left putamen in $12 \mathrm{MR}$ brain images. By using PCA, we can build a model of the shape-intensity profile of the left putamen. Figure 2 illustrates zero level sets and the associated intensities corresponding to the mean and three primary modes of variance of the distribution of the profile of the left putamen. Note that the mean shape-intensity pair and primary modes appear to be reasonable representative shapes and appearances being learned. The shape varies correspondingly as the associated gray levels vary, and vice versa.

We will then add some regularizing terms[9] into our active contour model: a general boundary smoothness prior, $p_{B}(S)=e^{-\mu \oint_{S} d s}$, and a prior for the size of the region, $p_{A}(S)=e^{-\nu A^{c}}$, where $A$ is the size of the region of shape $S, c$ is a constant and $\mu$ and $\nu$ are scalar factors. Thus, the prior joint probability $p(S, I)$ in equation (1) can be approximated by a product of the following probabilities:

$$
p(S, I)=p(\alpha) \cdot p_{B}(S) \cdot p_{A}(S)
$$



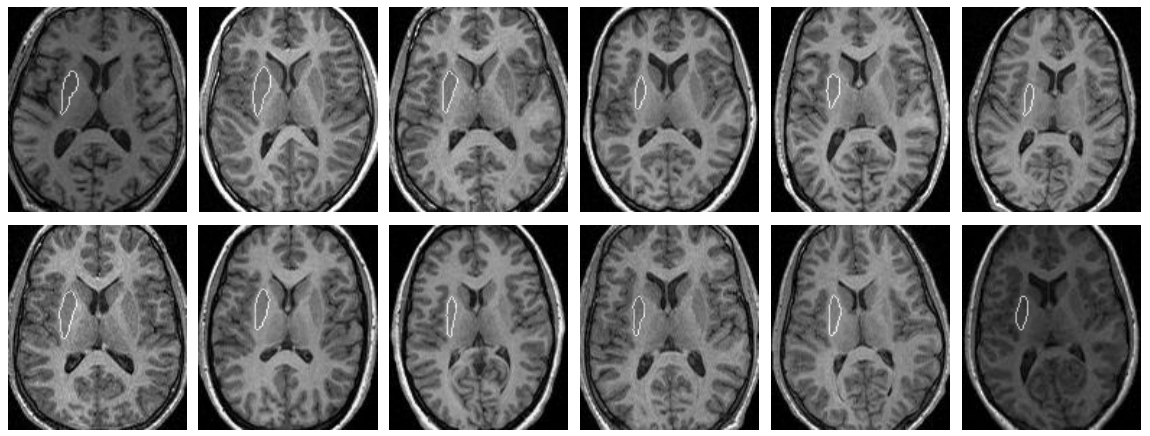

Fig. 1. Training set:outline of left putamen in 12 2D MR brain images.

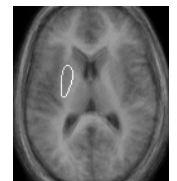

(1)

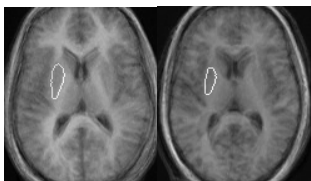

(2)

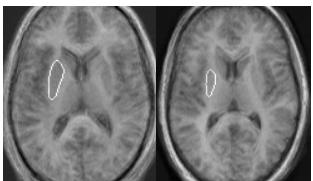

(4)
(5)

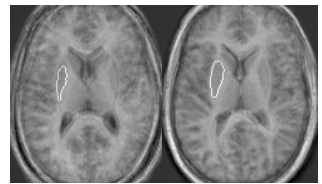

(6)
(7)

Fig. 2. The three primary modes of variance of the left putamen and the image gray levels. (1):The mean; (2),(3): $\pm \sigma$ variance of the $1^{\text {st }}$ primary mode; (4),(5): $\pm \sigma$ variance of the $2^{\text {nd }}$ primary mode; (6),(7): $\pm \sigma$ variance of the $3^{\text {rd }}$ primary mode.

Notice that the MAP estimation of the shape in equation (1), $\hat{S}$, is also the minimizer of the energy functional $E$ shown below in equation (5). This minimization problem can be formulated and solved using the level set method.

$$
\begin{aligned}
E(S) & =-\ln p(S / I) \propto-\ln p\left(S, I=I_{0}\right)=-\ln \left[p(\alpha) \cdot p_{B}(S) \cdot p_{A}(S)\right] \\
& \propto \frac{1}{2} \alpha^{T} \Sigma_{k}^{-1} \alpha+\mu \oint_{S} d s+\nu A^{c}
\end{aligned}
$$

\subsection{Level Set Formulation of the Model}

We will minimize the energy term $E(S)$ and we will add image gray level based energy terms defined by Chan [7]. Thus, the energy functional of shape $S$ is:

$$
\begin{aligned}
E_{\text {total }}\left(c_{1}, c_{2}, S\right)= & E(S)+\lambda_{1} \int_{(x, y, z) \text { inside }(S)}\left|I(x, y, z)-c_{1}\right|^{2} d x d y d z \\
& +\lambda_{2} \int_{(x, y, z) \text { outside }(S), \text { inside }\left(\Omega_{S}\right)}\left|I(x, y, z)-c_{2}\right|^{2} d x d y d z
\end{aligned}
$$

where $\lambda_{1}$ and $\lambda_{2}$ are non-negative fixed parameters. Constants $c_{1}, c_{2}$, depending on $S$, are the averages of $I$ inside $S$ and respectively outside $S$ but also inside a certain domain $\Omega_{S}$ that contains $S$. Therefore, our method is to minimize the above energy $E_{\text {total }}\left(c_{1}, c_{2}, S\right)$.

In the level set method, $S$ is the zero level set of a higher dimensional surface $\psi$, i.e., $S=\{(x, y, z) \mid \psi(x, y, z)=0\}$. The evolution of surface $S$ is given by the zero-level surface at time $t$ of the function $\psi(t, x, y, z)$. 
For the level set formulation of our model, using the technique developed in [7], we replace $S$ with $\psi$ in the energy functional in equation (6) using regularized versions of the Heaviside function $H$ and the Dirac function $\delta$, denoted by $H_{\varepsilon}$ and $\delta_{\varepsilon}[7]$ (described below):

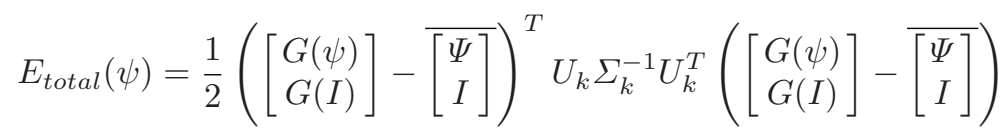

$$
\begin{aligned}
& +\mu \int_{\Omega} \delta_{\varepsilon}(\psi(x, y, z))|\nabla \psi(x, y, z)| d x d y d z \\
& +\nu \int_{\Omega}\left(1-H_{\varepsilon}(\psi(x, y, z))\right) d x d y d z \\
& +\lambda_{1} \int_{\Omega}\left|I(x, y, z)-c_{1}\right|^{2}\left(1-H_{\varepsilon}(\psi(x, y, z))\right) d x d y d z \\
& +\lambda_{2} \int_{\Omega_{S}}\left|I(x, y, z)-c_{2}\right|^{2} H_{\varepsilon}(\psi(x, y, z)) d x d y d z
\end{aligned}
$$

where $\Omega$ denotes the image domain. $G(\cdot)$ is an operator to form the column vector (as described in section 2.2) of a matrix by column scanning. $g(\cdot)$ is the inverse operator of $G(\cdot)$. To compute the associated Euler-Lagrange equation for $\psi$, we minimize $E_{\text {total }}$ with respect to $\psi$. Parameterizing the descent direction by artificial time $t \geq 0$, the evolution equation in $\psi(t, x, y, z)$ is:

$$
\begin{aligned}
\frac{\partial \psi}{\partial t}= & \delta_{\varepsilon}(\psi)\left[\mu \cdot \operatorname{div}\left[\frac{\nabla \psi}{|\nabla \psi|}\right]+\nu+\lambda_{1}\left|I-c_{1}\right|^{2}-\lambda_{2}\left|I-c_{2}\right|^{2}\right] \\
& -g\left(U_{k 1} \Sigma_{k}^{-1}\left[U_{k 1}^{T}(G(\psi)-\bar{\Psi})+U_{k 2}^{T}(G(I)-\bar{I})\right]\right)
\end{aligned}
$$

where $U_{k 1}$ and $U_{k 2}$ are the upper and lower half of the matrix $U_{k}$ respectively, i.e., $\left[U_{k}\right]=\left[\begin{array}{l}U_{k 1} \\ U_{k 2}\end{array}\right] \cdot H_{\varepsilon}(z)=\frac{1}{2}\left[1+\frac{2}{\pi} \arctan \left(\frac{z}{\varepsilon}\right)\right], \delta_{\varepsilon}(z)=\frac{\varepsilon}{\pi\left(\varepsilon^{2}+z^{2}\right)}[9] . c_{1}(\psi)=$ $\frac{\int_{\Omega} I(x, y, z) \cdot(1-H(\psi(x, y, z))) d x d y d z}{\int_{\Omega}(1-H(\psi(x, y, z))) d x d y d z}, c_{2}(\psi)=\frac{\int_{\Omega_{S}} I(x, y, z) \cdot H(\psi(x, y, z)) d x d y d z}{\int_{\Omega_{S}} H(\psi(x, y, z)) d x d y d z}[9]$.

\subsection{Evolving the Surface}

Given the surface $\psi$ at time $t$, we seek to compute the evolution steps that bring the zero level set to the correct final segmentation based on the shapeappearance joint prior information and image information. We first set up $p(\alpha)$ from the training set using PCA. At each stage of the algorithm, we recompute the constants $c_{1}\left(\psi^{t}\right)$ and $c_{2}\left(\psi^{t}\right)$, then update $\psi^{t+1}$. This is repeated until convergence.

\section{$3 \quad$ Experimental Results}

We have used our model on various medical images. In our experiments, we generally choose the parameters as follows: $\lambda_{1}=\lambda_{2}=\lambda, \mu=0.00005 \cdot 255^{2}$, $\nu=0$. All the tested images are not in their training sets. 

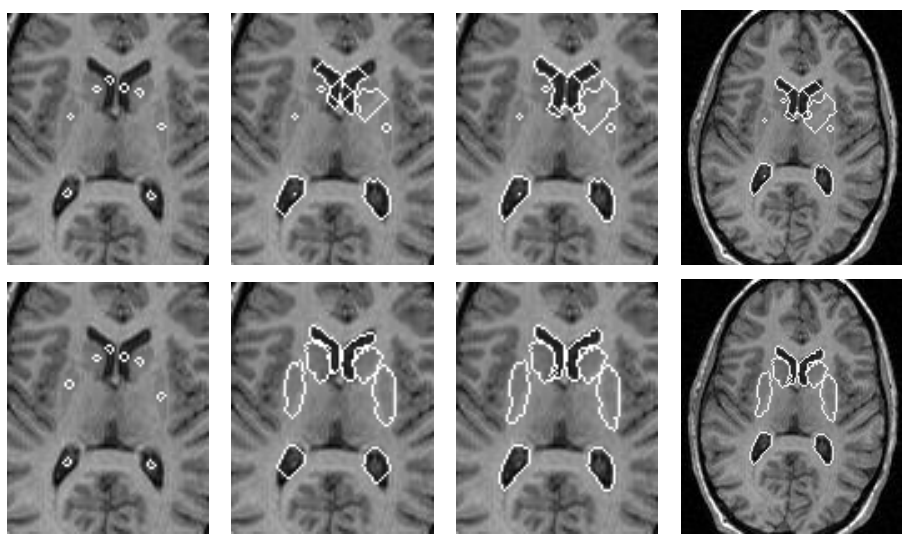

Fig. 3. Segmentation of 8 sub-cortical structures (the lateral ventricles $(\lambda=1)$, heads of the caudate nucleus $(\lambda=0.3)$, and putamina $(\lambda=0.2))$ in a MR brain image without prior information (top) and with shape-appearance joint prior (bottom).

We first consider a 2D MR brain image with eight sub-cortical structures of different intensities and with blurred boundaries. Figure 3 top shows a few steps of the segmentation using only gray level information. Only the lower (posterior) portions of the lateral ventricles can be segmented perfectly since they have clearer boundaries. Figure 3 bottom shows the results of using our shapeappearance joint prior model, the training set model of the left putamen is shown in Figures 1 and 2. The curves are able to converge on the desired boundaries even though some parts of the boundaries are too blurred to be detected using only gray level information. The segmentation converges in several minutes on an SGI Octane with a $255 \mathrm{MHz}$ R10000 processor.

We then test our method using 3D images. Figure 4 shows a few steps in the segmentation of the left hippocampus in a MR brain image. Figure 5 illustrates 3 steps in the segmentation of the left amygdala. Segmentating the two structures

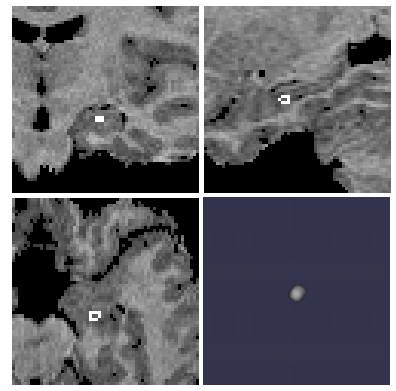

Initial

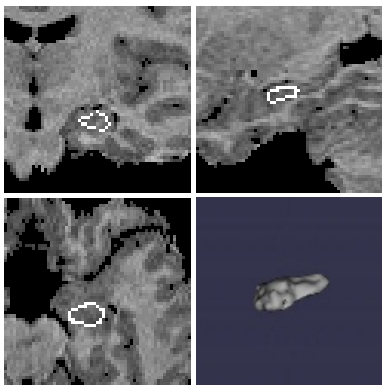

Middle

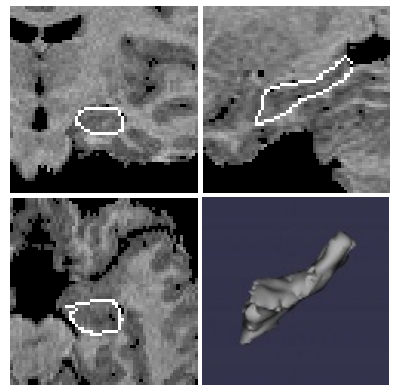

Final

Fig. 4. Segmentation of the left hippocampus. Three orthogonal slices and the 3D surfaces are shown for each step. The training set consists of $12 \mathrm{MR}$ images. $\lambda=0.01$. 


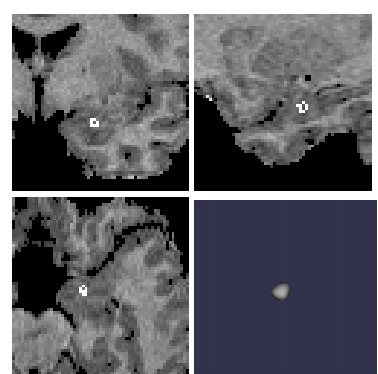

Initial

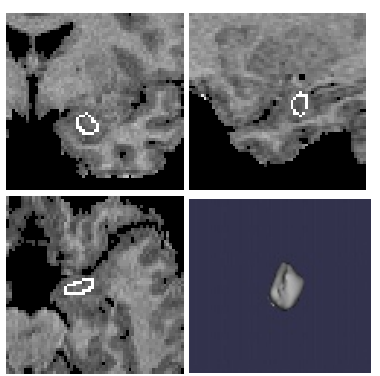

Middle

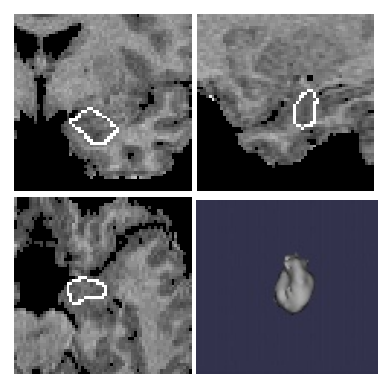

Final

Fig. 5. Segmentation of the left amygdala. Three orthogonal slices and the 3D surfaces are shown for each step. The training set consists of $12 \mathrm{MR}$ brain images. $\lambda=0.01$.

can be very tough without using prior information since both structures have very poorly defined boundaries. After using our shape-appearance joint prior model, as shown in Figure 4 and 5, the two structures can be clearly segmented.

To validate the segmentation results, we test our model on 12 different images for each of the above 3 cases respectively, the tested images are not in their training sets. We then compute the undirected distance of pixels between the boundary of the computed segmentation $A\left(N_{A}\right.$ points) and the boundary of the manual segmentation $B: H(A, B)=\max (h(A, B), h(B, A)), h(A, B)=$ $\frac{1}{N_{A}} \sum_{a \in A} \min _{b \in B}\|a-b\|$. For our experiments, the mean distances show improvement in all the 3 cases comparing with/without the shape-appearance prior: average sub-cortical structures $2.0 \mathrm{~mm} / 9.2 \mathrm{~mm}$, hippocampus $1.8 \mathrm{~mm} / 11.2 \mathrm{~mm}$, amygdala $1.6 \mathrm{~mm} / 5.8 \mathrm{~mm}$. Virtually all the boundary points obtained using our model lie within one or two voxels of the manual segmentation.

We also test the robustness of our algorithm to noise. We add Gaussian noise to the MR image in Figure 3(the mean intensities of white/gray matters: 45/65), then segment it. Figure 6 shows the segmentation results with Gaussian noise of standard deviation of 20(top) and 40(bottom). Figure 7 shows the segmentation errors of the lower portion of the left lateral ventricle in three cases: with no prior, with shape prior, and with shape-appearance joint prior. As the variance
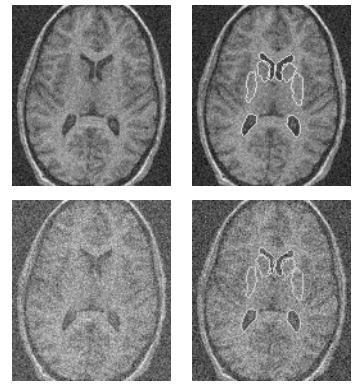

Fig. 6. Original and segmented images with Gaussian noise of $\sigma=$ 20 top) and 40 (bottom).

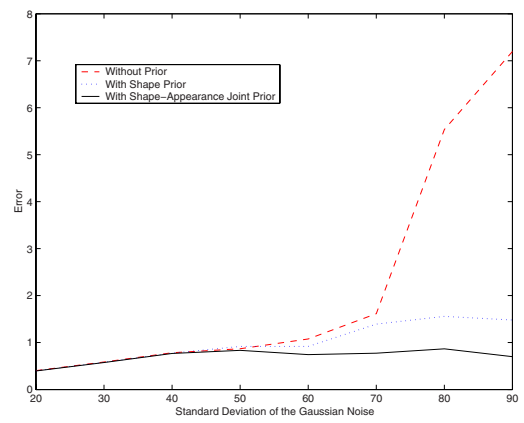

Fig. 7. Segmentation errors(unit:mm) with different variances of Gaussian noise. 
of the noise goes up, the error for no prior increases rapidly since the structure is too noisy to be detected using only gray level information. However, for the methods with shape prior and with shape-appearance joint prior, the errors are much lower and are locked in a very small range even when the variance of the noise is very large. Note that our shape-appearance joint prior model achieves the smallest error among all the cases.

\section{Conclusions}

In this paper, we proposed a MAP estimation framework based model for image segmentation using shape-appearance joint prior information. We introduce a representation for the shape-intensity joint density function, and define joint probability distribution over the variations of the shape-intensity pair in a set of training images. We estimate the MAP shape of the object using evolving level set based on the associated Euler-Lagrange equation. By using level set representation of the shape, we avoid the need for point correspondences during the training phase. From various experiments on 2D/3D medical images, we have demonstrated that our model is able to handle multidimensional data and robust to noise. This approach can be used to segment images with poor image contrast, noise, and missing or diffuse boundaries. As a powerful approach to deformable models, the MAP framework is flexible to combine prior information and given image information to realize image segmentation. While we have not pursued in this paper, a more unified model could be used to incorporate shape priors, neighbor constraints[8][9] as well as image gray level priors.

\section{References}

1. M. Kass, A. Witkin, D. Terzopoulos.: Snakes: Active contour models. Int'l Journal on Computer Vision, 1 (1987) 321-331.

2. S. Osher and J. A. Sethian.: Fronts propagating with curvature-dependent speed: Algorithms based on Hamilton-Jacobi Formulation. J. Comp. Phy., 79 (1988) 1249.

3. L.Staib, J. Duncan.: Boundary finding with parametrically deformable models. PAMI, 14(11) (1992) 1061-1075.

4. M. Leventon, E. Grimson, and O. Faugeras.: Statistical shape influence in geodesic active contours. IEEE Conf. on Comp. Vision and Patt. Recog., 1 (2000) 316-323.

5. T.F. Cootes, A. Hill, C.J. Taylor, and J. Haslam.: Use of active shape models for locating structures in medical images. Image and Vision Computing, 12(6): 355-365, July 1994.

6. T. Cootes, C. Beeston, G. Edwards, and C. Taylor.: Unified framework for atlas matching using active appearance models. IPMI, (1999).

7. T. Chan, L. Vese.: Active Contours Without Edges. IEEE Transactions on Image Processing, vol.10 No. 2 (2001) 266-277.

8. J. Yang, L. Staib and J. Duncan: Statistical Neighbor Distance Influence in Active Contours. MICCAI, vol.1 (2002) 588-596.

9. J. Yang, L. Staib and J. Duncan: Neighbor-Constrained Segmentation with 3D Deformable Models. IPMI, (2003) 198-209. 